\title{
Influence of Uniaxial [110] Stress on the Silicon Conduction Band Structure: Stress Dependence of the Nonparabolicity Parameter
}

\author{
V.A. Sverdlov, G. Karlowatz, E. Ungersboeck, and H. Kosina \\ Institute for Microelectronics, TU Wien, 1040 Vienna, Austria \\ \{sverdlov|karlo|ungersboeck|kosina\}@iue.tuwien.ac.at
}

\begin{abstract}
An alytical expression for the dependence of the nonparabolicity parameter on shear stress is presented. At $3 \mathrm{GPa}$ the nonparabolicity parameter is shown to increase by a factor of 1.7. Stress dependence of the nonparabolicity parameter is verified by comparing the density-of-states obtained analytically and from the empirical pseudopotential method, and good agreement is found. Increase in the nonparabolicity parameter increases the after-scattering density-of-states and hence the scattering rates, which results in a $25 \%$ suppression of the mobility enhancement due to effective mass decrease in a $3 \mathrm{~nm}$ thin body FET at $3 \mathrm{GPa}[110]$ stress.
\end{abstract}

\section{Model}

Stress-induced mobility engineering has become a key technique to increase the performance of modern CMOS devices [1]. Inherent to [110] uniaxial stress, shear distortion induces pronounced modifications in both the longitudinal [2, 3] and transversal [1, 2, 3, 4] effective masses. However, a possible stress dependence of the nonparabolicity parameter has not yet been considered.

The nonparabolicity of the lowest conduction band is due to the presence of the second conduction band [5]. At the band minimum the gap between the two bands is $\Delta=0.5$ $\mathrm{eV}$. The theoretical nonparabolicity parameter $\alpha$ was found to be consistent with the value $\alpha_{0}=0.5 \mathrm{eV}^{-1}$ extracted experimentally. Shear strain $\varepsilon_{x y}\left(\varepsilon_{x y}>0\right.$ for [110] tensile stress) lifts the degeneracy of the two lowest conduction bands at the $X$ point of the Brillouin zone in [001] valleys [4]. This results in a shift of the conduction band minimum both in energy and distance with respect to the $\mathrm{X}$ point (Fig.1). The transversal effective mass $m_{t} \mathrm{f}$ the [001] valleys becomes anisotropic and develops two branches $m_{t 1}, m_{t 2}$ as shown in Fig.2. Shear stress also affects the nonparabolicity parameter $\alpha$ of the [001] valleys. Introducing new variables

$$
\tilde{k}_{x}=p_{x} / \sqrt{m_{t 2}(\eta)}, \tilde{k}_{y}=p_{y} / \sqrt{m_{t 1}(\eta)}
$$

where $\eta=2 D \varepsilon_{x y} / \Delta$ is the normalized shear strain, with $D$ being the deformation potential for shear strain [4], one separates the renormalization due to stress of the parabolic 


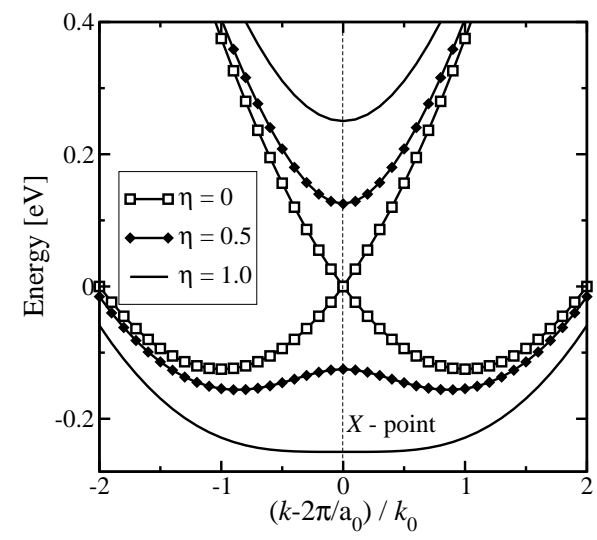

Figure 1: Conduction band profi le along [001] direction for different stress values in [110] direction.

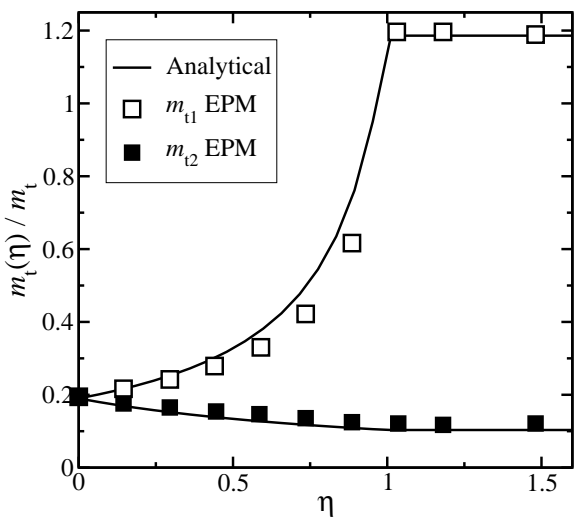

Figure 2: Dependences of the transversal effective mass on shear stress. The mass develops two branches.

part of the density-of-states (DOS) from the nonparabolic contribution. Proceeding in analogy to the Appendix B of [5], one arrives at the following dispersion relation

$$
E\left(\tilde{k}_{x}, \tilde{k}_{y}\right)=\frac{\hbar^{2}\left(\tilde{k}_{x}^{2}+\tilde{k}_{y}^{2}\right)}{2}-\left(m_{t 2}(\eta) \tilde{k}_{x}^{2}-m_{t 1}(\eta) \tilde{k}_{y}^{2}\right)^{2} \frac{\hbar^{4}}{2 M^{2} \Delta},
$$

where $M$ is a parameter of the $\mathbf{k} \mathbf{p}$ theory, $M \quad 1.2 m_{t}$. The last term gives the nonparabolicity correction to the density of states. Assuming the last term to be small, we compute the correction to the DOS and identifying it with the isotropic nonparabolicity parameter $\alpha(\eta)$.

\section{Results}

Taking into account the dependences of masses $m_{t 1}(\eta)$ and $m_{t 2}(\eta)$ on stress $\eta[2,3]$ and proceeding similar to the Appendix B of [5], we arrive at an expression for the strain dependent nonparabolicity parameter $\alpha(\eta)$ :

$$
\alpha(\eta)=\alpha_{0} \frac{1+2\left(\eta m_{t} / M\right)^{2}}{1-\left(\eta m_{t} / M\right)^{2}} .
$$

Expression (2) is plotted in Fig.3. The nonparabolicity parameter depends on the suqare of the shear stress value and therefore not on the sign. Due to the quadratic dependence on shear stress, the relative increase of $\alpha(\eta)$ is more important at relatively large stress values.

Fig.4 illustrates the density-of-states of the [001] valley as a function of energy relative to the minimum, obtained numerically from empirical pseudo-potential calculations, for the unstressed case and stress equal to $3 \mathrm{GPa}$. According to (2), the value of the nonparabolicity parameter at $3 \mathrm{GPa}$ is $70 \%$ higher than the unstrained value $\alpha_{0}$. The DOS is normalized to the analytical DOS (dotted lines) computed with strain dependent transversal effective masses $m_{t 1}(\eta), m_{t 2}(\eta)$ and strain dependent longitudinal mass $m_{l}(\eta)$. Without the nonparabilicity parameter included, considerable deviations in the 


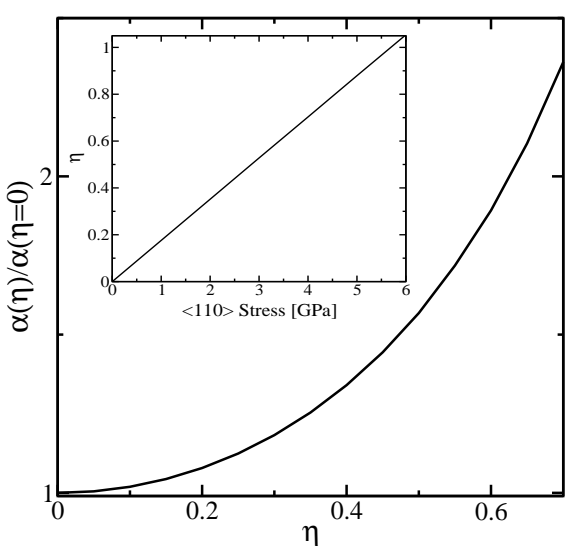

Figure 3: Analytical expression for dependence of the nonparabolicity parameter on stress. Inset: relation between normalized stress and stress in GPa.

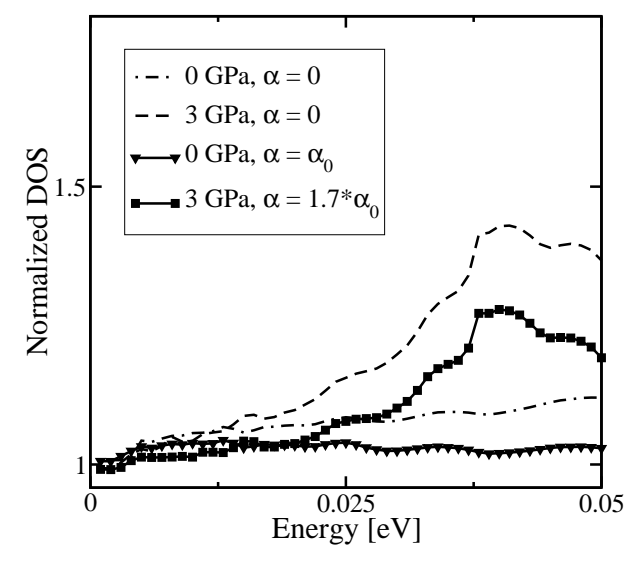

Figure 4: Numerical DOS in [001] valley normalized to the DOS analytical expressions obtained without (dotted lines) and with stress dependent nonarabolicity parameter (solid lines), for unstrained Si and Si under [110] uniaxial stress.

ratio of numerical DOS to its analytical value is observed at higher energies. The ratio becomes much closer to unity if the nonparabolicity correction due to stress dependent $\alpha(\eta)$ is taken into account in the analytical DOS (Fig.4, solid lines with symbols).

Deviation of the numerical DOS from the analytical model with stress dependent $\alpha$ is observed for larger energies at high stress value. The reason being that the energy difference between the valley minimum and the value at the $X$ point decreases with stress (Fig.1). A pronounced peak in the numerical DOS appears, which corresponds to the flat dispersion close to $X$. The energy value of the peak is shown in Fig. 5 as a function of shear stress and compared with the theoretical prediction. For stress values larger than $3 \mathrm{GPa}$ the energy difference from the minimum to the value at the $X$ point becomes smaller than $2 \times k_{B} T$, as seen in Fig.5, and a full-band description is required [2].

Through the modification of the density of the after-scattering states, the nonparabolicity parameter affects scattering rates, and therefore the electron mobility. As example we consider the mobility in a double-gate FET with a $3 \mathrm{~nm}$ thin Si body. The subband energies and corresponding wave functions are calculated from the Schrödinger equation coupled self-consistently with the Poisson equation for each value of the effective field. The wave functions are then used to calculate the scattering rates. Our transport calculations account for electron-phonon interactions $[5,6]$ and surface roughness scattering, which are the dominant mechanisms determining the mobility in the region of high effective fields. We use the original formulation by Prange and Nee [7, 8, 9] for the surface roughness scattering matrix elements.

Results of the mobility simulations in an ultra-thin body FET with strain along the [110] direction, with and without stress dependence of the nonparabolicity parameter taken into account, are shown in Fig. 6 together with the mobility in an unstrained SOI. Due to stress dependence of the the transversal mass the mobility in UTB under uniaxial stress is significantly enhanced. However, when the stress dependence of the nonparabolicity 
parameter is included, the mobility enhancement is suppressed by almost $25 \%$ in a $3 \mathrm{~nm}$ thin SOI FET at a stress level of $3 \mathrm{GPa}$ (Fig.6).

\section{Conclusion}

An alytical expression for the stress dependence of the nonparabolicity parameter is verified by comparing the corresponding DOS to the numerical DOS obtained from the empirical pseudo-potential method. The stress dependence of the nonparabolicity parameter results in almost $25 \%$ decrease of the mobility enhancement in a $3 \mathrm{~nm}$ thin SOI FET at high stress level.

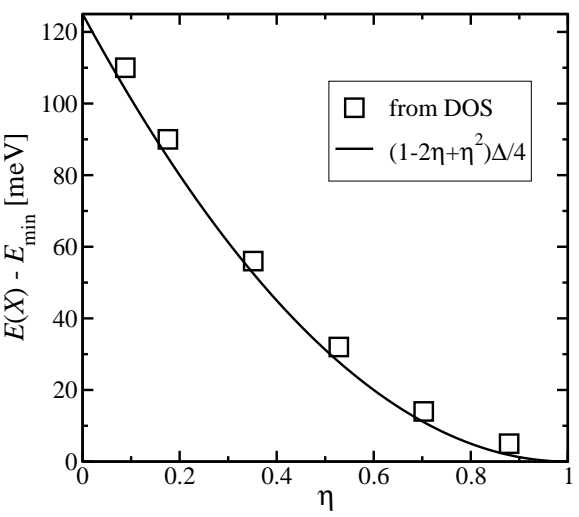

Figure 5: Energy at the $X$ point relative to the valley minimum: analytical results and EPM data.

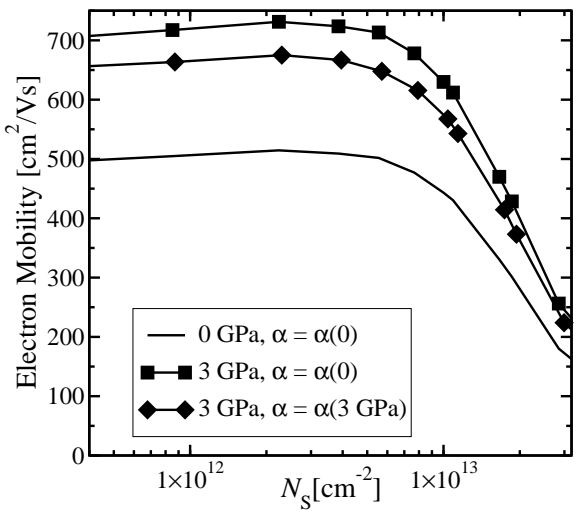

Figure 6: Mobility in a $3 \mathrm{~nm}$ thin SOI FET at $3 \mathrm{GPa}$ [110] stress computed with and without stress dependence of the nonparabolicity parameter.

\section{Acknowledgments}

This work, as part of the European Science Foundation EUROCORES Programme FoNE, was supported by funds from FWF (project I79-N16), CNR, EPSRC and the EC Sixth Framework Programme, under Contract N. ERAS-CT-2003-980409.

\section{References}

[1] K. Uchida, T. Krishnamohan, K. C. Saraswat, and Y. Nishi, in IEDM Techn. Dig. (2005), pp. $129-132$.

[2] E. Ungersboeck et al., IEEE Trans.Electron Devices (in print, 2007).

[3] V. Sverdlov, E. Ungersboeck, H. Kosina, and S. Selberherr, in Proc. EUROSOI 2007 (January, 2007), pp. 39-40.

[4] J. C. Hensel, H. Hasegawa, and M. Nakayama, Phys. Rev. 138, A225 (1965).

[5] C. Jacoboni and L. Reggiani, Reviews of Modern Physics 55, 645 (1983).

[6] P. J. Price, Ann.Phys. 133, 217 (1981).

[7] R. E. Prange and T. W. Nee, Physical Review 168, 779 (1968).

[8] M. V. Fischetti et al., J.Appl.Phys. 94, 1079 (2003).

[9] D. Esseni, IEEE Trans.Electron Devices 51, 394 (2004). 